Background: We present the first report of high-titer autoantibodies in NLRP3-associated autoinflammatory disease(NLRP3-AID).Because systemic autoinflammatory disease(SAID) is characterised by the lack of autoreactive T-cells or autoantibodies, we made a systemic review on the theme of autoantibody in SAID to clarify this phenomenon.

Objectives: We present the first report of high-titer autoantibodies NLRP3-AID, and discuss autoantibody in classical SAID.

Methods: We collected the clinical data of the patient with NLRP3-AID who had high-titer autoantibodies,and made a systemic review about autoantibody in SAID.

Results: A 38-year-old Chinese Han patient was definitely diagnosed as NLRP3-AID because of cold-triggered urticaria-like rash and fever,arthralgia,binaural sensorineural deafness,chronic meningitis, high inflammatory marker and de novo NLRP3 T348M variant. Figure 1 shows pedigree of the patient. Meanwhile,she had positive antinuclear antibody(ANA) with a nucleolar pattern of 1:160, positive anti- $\beta 2 \mathrm{GPI}$ antibody $54-68 \mathrm{AU} / \mathrm{ml}$ (normal range $<20 \mathrm{AU} / \mathrm{ml}$ ) and positive lupus anticoagulant 1.24-1.29 (normal range $\leq 1.2$ ). Literature review found that 13 articles reported autoantibodies in Familial Mediterranean fever (FMF), and there was no autoantibody reported in hyperimmunoglobulinemia D syndrome (HIDS),TNF receptor-associated periodic syndrome(TRAPS) and NLRP3-AID. The prevalence of ANA,anti-dsDNA,RF and anti-CCP in patients with FMF was similar to healthy controls.

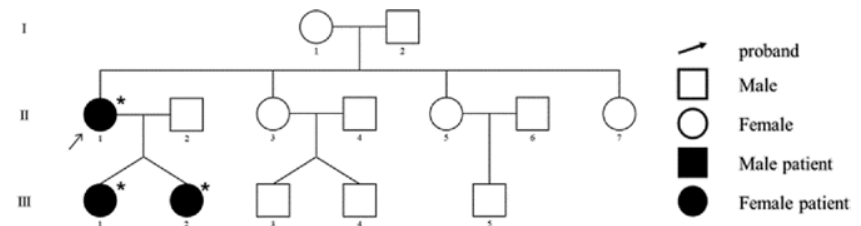

Fig. 1. Pedigree of the patient. The black arrow indicates the proband. The asterisks indicate the individuals who had clinical manifestations and carry the NLRP3 T348M variant.

Conclusion: Patients with NLRP3-AID can have high-titer ANA and APLs by accident. If patients with high-titer autoantibodies have characteristic manifestations of SAIDs instead of typical features of autoimmune diseases, we should make the final diagnosis through detailed investigation and genetic testing. References:

[1] Ben-Chetrit E, Gattorno M, Gul A, et al. Consensus proposal for taxonomy and definition of the autoinflammatory diseases (AIDs): a Delphi study. Annals of the rheumatic diseases 2018; 77(11): 1558-65.

[2] Kuemmerle-Deschner JB, Ozen S, Tyrrell PN, et al. Diagnostic criteria for cryopyrin-associated periodic syndrome (CAPS). Annals of the rheumatic diseases 2017; 76(6): 942-7.

Disclosure of Interests: None declared

DOI: 10.1136/annrheumdis-2020-eular.5670

\section{AB1278 $\quad$ A CASE REPORT OF TOCILIZUMAB INDUCE RAPID REMISSION OF ADULT-ONSET STILL'S DISEASE WITH LIFE-THREATENING INTERSTITIAL LUNG DISEASE AND MACROPHAGE ACTIVATION SYNDROME}

G. Zhang ${ }^{1}$, W. Liu ${ }^{1}$, T. Xu ${ }^{1}$, X. Zhang ${ }^{1} .{ }^{1}$ Guangdong Provincial People's Hospital/Guangdong Academy of Medical Sciences, Rheumatology, Guangzhou, China

Background: Adult-onset still's disease (AOSD) is a systemic inflammatory condition characterized by fevers, polyarthritis, skin rashes and increased leukocyte and neutrophil counts [1]. Sometimes life-threatening complications such as macrophage activation syndrome (MAS), disseminated intravascular coagulopathy (DIC) can be occurred without response to glucocorticoid and disease-modifying antirheumatic drugs (DMARDs).

Objectives: To describe an AOSD patient with life-threatening interstitial lung disease (ILD) and MAS response to tocilizumab.

Methods: This is a case report at our medical practice.

Results: A 43-year-old man was hospitalized for a 2-week history of fever accompanying skin rashes, polyarthritis, sore throat, leukocytosis (the maximum leukocyte count $37.25 \times 10^{9} / \mathrm{L}$ with $89.9 \%$ neutrophils), elevated levels of C-reactive protein $(190.9 \mathrm{mg} / \mathrm{L})$ and ferritin level was very high $(>15000 \mathrm{ng} / \mathrm{ml})$, Several antibiotics were consequently used, but fever was on going. Chest CT displayed diffuse interstitial lung disease. Infectious diseases and lymphoma were excluded and AOSD with ILD was diagnosed. However, MAS occurred the 20 days later after the pulse of intravenous MP (MP $500 \mathrm{mg} \times 3$ days) following MP $40 \mathrm{mg} /$ day, which cannot stop the situation deteriorating. The minimum total white blood cells, hemoglobin and platelets went down to $0.25 \times 10^{9} / \mathrm{l}, 53 \mathrm{~g} / \mathrm{l}$ and $18 \times 10^{9} /$. Finally, tocilizumab rescued this patient from desperation.

Conclusion: Tocilizumab was effective in AOSD with life-threatening ILD and MAS

\section{References:}

[1] E. Feist, S. Mitrovic, and B. Fautrel, Mechanisms, biomarkers and targets for adult-onset Still's disease. Nat Rev Rheumatol 14 (2018) 603-618.

Disclosure of Interests: None declared

DOI: 10.1136/annrheumdis-2020-eular.2529

\section{HPR Measuring health (development and measure- ment properties of PROs, tests, devices)}

\section{AB1279-HPR A DESCRIPTIVE STUDY RELATED TO THE ADHERENCE BEFORE AND AFTER ENROLLING IN A MULTIDISCIPLINARY EDUCATIONAL PROGRAM}

D. Buitrago-Garcia ${ }^{1}$, F. Rodriguez ${ }^{2}$, G. Sánchez ${ }^{1}$, P. Santos-Moreno ${ }^{3}$.

${ }^{1}$ Fundación Universitaria de Ciencias de la Salud, Epidemiology, Bogota, Colombia; ${ }^{2}$ Biomab - Center for Rheumatoid Arthritis, Patient Education

Program, Bogota, Colombia; ${ }^{3}$ Biomab - Center for Rheumatoid Arthritis, Rheumatology, Bogota, Colombia

Background: Rheumatoid arthritis (RA) is an inflammatory, chronic disease. It leads to deformity and destruction of joints through the erosion of cartilage and bone. Patients with RA report to suffer symptoms in hands, joints, swelling, loss of motion, muscle weakness among others.(1).Centers of excellence in RA have proposed a multidisciplinary model of care with an initial diagnosis, treatment prescription and follow-up with a rheumatologist, periodic consultations with a physiatrist, psychologist, physiotherapist, occupational therapy, nutrition and a patient focused program(2). With this model of care, the patient is seen as a whole, and the expectation is to achieve the best results in the management of RA. However, if the patient does adhere the model becomes ineffective.

Objectives: The aim of this to report the attendance to a multidisciplinary model of care for patients with RA that attend to a specialized center in Colombia, before and after enrolling in a educational program.

Methods: We performed a descriptive study. Patients enrolled our educational program in July 2019. In our institution patients are followed-up under T2T standards and a multidisciplinary approach, as part of our model of care they have periodic consultations with a rheumatology, physiatrist, psychologist, physiotherapist, occupational therapy and nutrition. We collected sociodemographic data, DAS28, and compare the attendance to each specialty at the beginning and at 6-month follow-up. Descriptive epidemiology was done, we calculated means, and standard deviations for continuous variables and categorical variables were presented as rates. We compared disease activity and adherence at the beginning of the program and after six months of attendance.

Results: We included 229 patients; mean age was 59 years \pm 10 ; $93 \%$ were female. At the beginning of our program, mean DAS28 was $2.57 \pm 1.19$, from all patients $65 \%$ were at remission, $11 \%$ at low disease activity $19 \%$ at moderate disease activity and, $5 \%$ at severe disease activity. Regarding adherence to our model, the medical specialty with the highest attendance was rheumatology (30\%) followed by, physical therapy (16\%) physiatrist consultation (15\%) psychology $(13 \%)$ and, occupational therapy $(11 \%)$; the specialty with the lowest attendance was nutrition ( $8 \%$ ). After six months of attendance to the educational program, we found an increasing number of patients in remission $67 \%$, low disease activity $15 \%$, moderate disease activity $18 \%$, we did not have patients with severe DA28. Regarding the medical specialties, we found a $3 \%$ rise in the attendance to the nutrition consultation and psychology consultation. We did no find statistical association between disease activity and adherence to the model. Conclusion: These results are a clear example of how an educational program is capable of increasing awareness and improving the clinical outcomes and adherence to a multidisciplinary model for approaching RA. As other studies have shown(3), patient education interventions improve adherence to medication and to attendance to health care specialists.

References:

[1] Santos-Moreno P, Castaneda O, Garro B, Flores D, Sanchez G, Castro C. From the model of integral attention to the creation of centers of excellence in rheumatoid arthritis. Clinical rheumatology. 2015;34 Suppl 1:S71-7.

[2] Taibanguay N, Chaiamnuay S, Asavatanabodee P, Narongroeknawin P. Effect of patient education on medication adherence of patients with rheumatoid arthritis: a randomized controlled trial. Patient preference and adherence. 2019;13:119-29.

Acknowledgments: This project has been funded by a collaboration between the Ministry of Science, Technology and Innovation COLCIENCIAS (contract 746-2018), the Fundación Universitaria de Ciencias de la Salud and Biomab Center for Rheumatoid Arthritis

Disclosure of Interests: Diana Buitrago-Garcia: None declared, Fernando Rodriguez: None declared, GUILLERMO SÁNCHEZ: None declared, Pedro Santos-Moreno Grant/research support from: I have received research grants from Abbvie, Biopas-UCB, Janssen, Novartis, Pfizer., Speakers bureau: I have been a speaker for Abbvie, Biopas-UCB, Janssen, Lilly, Novartis, Pfizer, Roche, Sanofi. 


\section{AB1280-HPR REQUIRED FORCE TO OBTAIN A POSITIVE SQUEEZE TEST AUTOMATIZED IN PATIENTS WITH HAND ARTHRALGIA}

M. M. Castañeda-Martínez ${ }^{1}$, G. Figueroa-Parra ${ }^{1}$, D. Vega-Morales ${ }^{1}$, B. R. Vázquez Fuentes ${ }^{1}$, Y. G. Ordoñez Azuara ${ }^{2}$, J. A. Esquivel Valerio ${ }^{1}$, D. Á. Galarza-Delgado'. 'University Hospital "Dr. José Eleuterio González", Rheumatology Service, Monterrey, Mexico; ${ }^{2}$ University Hospital "Dr. José Eleuterio González", Family Medicine Service, Monterrey, Mexico

Background: Primary care physicians (PCP) are usually the first contact of people with inflammatory rheumatic diseases, and find the early symptoms of Rheumatoid Arthritis (RA) difficult to distinguish from those of other rheumatic diseases. A time-delay in the reference to Rheumatology is a health issue in several countries. The clinical aspects that general practitioner took into account in hand arthralgia patients are important to make the reference. In particular the Squeeze Test (ST) - which is simple to perform and rapidly done, ST is useful for identifying progression to RA in patients with undifferentiated arthritis. The ST has been described as not reliable because is clinician-dependent.

Objectives: To identify the required force that needs to be applied in order to obtain a positive Automatized Squeeze Test (AST) in a cohort of patients with hand arthralgia.

Methods: Ninety-seven patients were recruited in Family Medicine Consultation and in Rheumatology Consultation of the Hospital Universitario "Dr. José Eleuterio González" in Monterrey, Nuevo León, México. Eligible patients were adults (aged $\geq 18$ years) with hand arthralgia (that wasn't caused by trauma) as their chief complaint. After obtaining informed consent and after a questionnaire application, patients were submitted to AST maneuver, using an automated compressor with different forces already predetermined in the interface of the software used for compression.

Results: In this cohort of 98 patients, $79(80.6 \%)$ were women. The mean age was 51.14 years (SD 14.66). Ninety-six (97.9\%) patients were right handed. The diagnoses were Osteoarthritis (OA) $(16.3 \%)$, RA (5.1\%), Undifferentiated arthritis (1.2\%), Psoriatic arthritis (1.2\%) and Fibromyalgia (2\%). Force measures according to diagnoses are reported in Table 1.

Table 1. Diagnoses and mean forces

\begin{tabular}{lccc}
\hline Diagnosis & $\mathbf{n}(\%)$ & $\begin{array}{c}\text { Right hand force mean } \\
\mathbf{( k g / \mathbf { s } ^ { 2 } )} \mathbf{( S D )}\end{array}$ & $\begin{array}{c}\text { Left hand } \\
\text { force mean } \\
\mathbf{( k g / \mathbf { s } ^ { 2 } )}(\mathbf{S D})\end{array}$ \\
\hline OA & $16(16.3)$ & $3.53(2.74)$ & $3.18(2.73)$ \\
RA & $5(5.1)$ & $3.60(2.53)$ & $3.16(1.36)$ \\
UA & $1(1.2)$ & $7.60(0)$ & $8.70(0)$ \\
PsA & $1(1.2)$ & $7.60(0)$ & $7.80(0)$ \\
FM & $2(2.0)$ & $4.11(4.40)$ & $1.75(1.06)$ \\
\hline
\end{tabular}

OA, Osteoarthritis; RA, Rheumatoid Arthritis; UA, Undifferentiated Arthritis; PsA, Psoriatic Arthritis; FM, Fibromyalgia; SD, Standard Deviation

Conclusion: In the cases of RA and OA, the means of force to obtain a positive AST was lower than in the rest of the diagnoses.

References:

[1] Stack R, Nightingale P, Jinks C, Shaw K, Herron-Marx S, Horne R et al. Delays between the onset of symptoms and first rheumatology consultation in patients with rheumatoid arthritis in the UK: an observational study. BMJ Open. 2019;9(3):e024361.

Disclosure of Interests: None declared

DOI: 10.1136/annrheumdis-2020-eular.4515

\section{AB1281-HPR THE LEVELS OF VITAMIN D IN THE SPONDYLOARTHRITIS. DOES THE DEFICIT CORRESPOND TO THE INFLAMMATORY ACTIVITY?}

D. Castro-Corredor ${ }^{1}$, M. A. Ramírez Huaranga ${ }^{2}$, A. I. Rebollo Giménez ${ }^{2}$, M. D. Mínguez Sánchez ${ }^{2}$, J. Anino-Fernández ${ }^{2}$, I. M. De Lara Simón ${ }^{3}$, J. L. Cuadra Díaz ${ }^{2}{ }^{1}$ Hospital General Universitario Ciudad Real, Ciudad Real, Spain; ${ }^{2}$ Hospital General Universitario Ciudad Real, Rheumatology Department, Ciudad Real, Spain; ${ }^{3}$ Hospital General Universitario Ciudad Real, Pathology Department, Ciudad Real, Spain

Background: Spondyloarthritis is a group of chronic inflammatory diseases with involvement of the axial skeleton (mainly), and also of peripheral joints. Patients with spondyloarthritis have a significant prevalence of vitamin $\mathrm{D}$ levels below normal and that would correlate with the degree of activity of the disease.
Objectives: To determine the association between vitamin $D$ deficiency and the degree of activity of the disease (inflammatory activity) in a cohort of patients with spondyloarthritis.

Methods: Case-control type analytical observational study. We propose a retrospective review of the database of patients with spondyloarthritis (according ASAS2010 criteria) who were treated in the outpatient clinics of the Rheumatology Service of the General University Hospital of Ciudad Real during June 2018 to June 2019. Patients with the data will be selected. necessary for the analysis of the variables under study. The numerical variables of normal distribution evaluated will be described using measures of frequency and measures of central tendency / dispersion as appropriate. To assess the association between vitamin $\mathrm{D}$ levels and activity index, the odds ratio (OR) is calculated, with a $95 \%$ confidence level and the T-student for related samples.

Results: The final results of the study are presented. 115 patients were analyzed, of which 64 were men and 51 women, with an average age of 45.97 years (+/- 13.41 DE). $47 \%$ were ankylosing spondylitis, $21 \%$ psoriatic arthropathy, $16 \%$ undifferentiated spondyloarthritis, $7 \%$ spondyloarthropathy associated with inflammatory bowel disease and $9 \%$ were spondyloarthropathy associated with inflammatory bowel disease. The average of the activity was a BASDAI of 4.57 (+/- 2.35 SD) and measured by DAPSA was 12.61 (+/- 6.76 SD). 63 and 14 patients had activity measured by BASDAI and DAPSA, respectively. $49.56 \%$ patients presented an elevation of acute phase reactants. Vitamin $D$ levels were 23.81 (+/- 10.5 SD). $77.4 \%$ presented figures of vitamin D deficiency or insufficiency. When performing the association analysis, the vitamin $D$ deficit / insufficiency presented an OR $10(95 \% \mathrm{Cl}$ : 3.66-27.29, $\mathrm{p}=<0.0001)$ with the degree of activity measured with BASDAI and DAPSA and against the elevation of RCP it was $3.63(95 \% \mathrm{Cl} 1.43-9.25, \mathrm{p}=0.0092)$ and against the elevation of ESR it was $2.76(95 \% \mathrm{Cl} 1.09-7,0, \mathrm{p}=0.0438)$. Regarding the comparative analysis of means between vitamin D deficiency/insufficiency and BASDAI/DAPSA it was +3.29 (95\% Cl: 1.34-8.09, $\mathrm{p}=0.0084)$.

Conclusion: Patients with spondyloarthritis, as in other autoimmune diseases, vitamin $D$ deficiency is associated with increased inflammatory activity (BASDAI, DAPSA, RCP and ESR), measured in different time periods. Therefore, an optimization of vitamin $D$ levels can imply an improvement in the patient's clinical situation, measured by both BASDAI and DAPSA, as well as by RCP and ESR. In addition, it is necessary to monitor bone mineral density due to the risk of fracture in these patients for their multietiology (corticosteroid treatments, biological FAMEs, inflammatory activity).

\section{References:}

[1] Lange U, Teichmann J, Strunk J, Müller-Ladner U, Schmidt KL. Association of 1.25 vitamin D3 deficiency, disease activity and low bone mass in ankylosing spondylitis. Osteoporos Int. 2005;16:1999-2004.

[2] Durmus B, Altay Z, Baysal O, Ersoy Y. Does vitamin D affect disease severity in patients with ankylosing spondylitis? Chin Med J. 2012;125:2511-2515.

[3] Mermerci Baskan B, Pekin Dogan y, Sivas F, Bodur H, Ozoran K. The relation between osteoporosis and vitamin $\mathrm{D}$ levels and disease activity in ankylosing spondylitis. Rheumatol Int. 2010;30:375-381.

Disclosure of Interests: None declared

DOI: 10.1136/annrheumdis-2020-eular.1637

\section{AB1282-HPR CONCORDANCE BETWEEN TUBERCULIN TEST AND INTERFERON-GAMMA RELEASE ASSAY IN THE SCREENING OF LATENT TUBERCULOSIS INFECTION IN PATIENTS WHO ARE GOING TO INITIATE A TNF INHIBITOR}

M. D. M. Cutillas Perez ${ }^{1}$, C. Marin Silvente ${ }^{1}$, E. Saiz ${ }^{1}$, M. F. Pina ${ }^{1} .{ }^{1}$ Meseguer General University Hospital Morales, Murcia, Spain

Background: The drugs that inhibit tumor necrosis factor (anti-TNF) alpha can reactivate a latent tuberculosis infection (ILTB) so requiring a rigorous screening before its onset. The tuberculin test (PT) has a high false negative rate in patients with immunomediated rheumatic diseases (IMID) and false positive in patients vaccinated with Bacillus Calmette Guérin (BCG). The neu methods of interferon gamma release (IGRA) seem to solve this problem, but its use is not standardized.

Objectives: Establish the degree of concordance in the diagnosis of ILTB between PT and IGRA in patients who are going to star an anti-TNF drug, in general, and in different situation like taking corticosteroids, being treated with disease modifying drugs, have been vaccinated with BCG or have risk factor for ILTB.

Methods: From May 2016 to November 2019, 195 patients with IMID who underwent ITLB screening prior to the initiation of an anti-TNF drug were included in this study. The concordance between PT and IGRA was calculated using the cohen's kappa index, for the general sample first and then for subgroups. An analysis of the factor that influence the result of PT and IGRA has also been carried out. 